Article

\title{
Measurement of Small-Slope Free-Form Optical Surfaces with the Modified Phase Retrieval
}

\author{
Xinxue Ma*, Jianli Wang, Bin Wang and Xinyue Liu \\ Changchun Institute of Optics, Fine Mechanics and Physics, Chinese Academy of Sciences, \\ Changchun 130033, China; wangjianli@ciomp.ac.cn (J.W.); eatingbeen@hotmail.com (B.W.); \\ liuxinyue@ciomp.ac.cn (X.L.) \\ * Correspondence: xinxuema@hotmail.com
}

check for updates

Citation: Ma, X.; Wang, J.; Wang, B.; Liu, X. Measurement of Small-Slope Free-Form Optical Surfaces with the Modified Phase Retrieval. Micromachines 2022, 13, 82. https:// doi.org $/ 10.3390 / \mathrm{mi1} 13010082$

Academic Editors: Lihua Li and W.B. Lee

Received: 18 November 2021 Accepted: 22 December 2021 Published: 4 January 2022

Publisher's Note: MDPI stays neutral with regard to jurisdictional claims in published maps and institutional affiliations.

Copyright: (C) 2022 by the authors. Licensee MDPI, Basel, Switzerland. This article is an open access article distributed under the terms and conditions of the Creative Commons Attribution (CC BY) license (https:// creativecommons.org/licenses/by/ $4.0 /)$.

\begin{abstract}
In this paper, we demonstrate the use of the modified phase retrieval as a method for application in the measurement of small-slope free-form optical surfaces. This technique is a solution for the measurement of small-slope free-form optical surfaces, based on the modified phase retrieval algorithm, whose essence is that only two defocused images are needed to estimate the wave front with an accuracy similar to that of the traditional phase retrieval but with less image capturing and computation time. An experimental arrangement used to measure the small-slope free-form optical surfaces using the modified phase retrieval is described. The results of these experiments demonstrate that the modified phase retrieval method can achieve measurements comparable to those of the standard interferometer.
\end{abstract}

Keywords: free-form; metrology; surfaces measurement; phase retrieval

\section{Introduction}

With the rapid development of national defense, aerospace, and other fields, the demand for high-precision and high-quality photoelectric products is increasing, and these photoelectric products are gradually developing toward miniaturization. Using free-form surfaces, the imaging quality of the optical imaging system can be greatly improved; the illumination uniformity of the optical illumination system can be evidently improved; and the transmission efficiency of the information transmission system can also be remarkably improved. With the recent advances in optical design and fabrication, the free-form optical surface is commonly used because of its better performance and compactness [1-3].

Because the free-form optical surface has more degrees of freedom for correcting optical aberrations, the high precision free-form optical surface metrology remains difficult and is still a challenge [4-6]. Therefore, in recent years, many scholars have studied the optical testing methods of free-form surfaces. Thus, a number of metrology methods have been developed [7-9], and these methods are roughly divided into contact metrology and non-contact metrology. Because it is easy to scratch the surfaces with contact metrology, non-contact metrology is preferred for high-precision optical surfaces. The recognized interferometer cannot measure the free-form surfaces. This is not only because the standard interferometry has some typical disadvantages, such as high sensitivity to vibrations or temperature fluctuations, hindering its usage to strictly controlled laboratory conditions, but also because the fringes are too dense, and the interference fringes cannot be generated.

In order to solve the problem of free-form optical surface metrology, we introduce a feasible non-contact measurement method called Phase Retrieval (PR), a high-precision method and an alternative to interferometry for optical testing, with the advantages of compactness, low-cost, and a stable system. PR has emerged as a potential solution for free-form surfaces metrology [10-13]. As PR is a method of wave-front sensing and a simple experimental arrangement in optical metrology, it has been used in system measurement and alignment; it has, for example, been used in the Hubble Space Telescope and the 
James Webb Space Telescope [14,15]. Besides, PR has also been applied to test spherical mirror surfaces and rotationally symmetric aspherical surfaces $[16,17]$. As we know, algorithm is the soul of PR. Some PR algorithms have robustness, multiple solutions, and stagnation; for example, the convergence speed of the fastest gradient search in the PR algorithm is not the fastest, and it usually falls into a local minimum. In addition, the conjugate gradient search method in the PR algorithm is more robust than the fastest gradient search method. Thus, we introduce a new PR algorithm, which potentially has the advantage of improving the efficiency of phase recovery in order to solve the limitations of the traditional PR in its iterative uncertainty and slow convergence speed $[18,19]$.

In this paper, we will first introduce the theory and the application of PR and then the improved PR algorithm in Section 2. In Section 3, the results and analysis of the experiment are presented. The conclusion is finally drawn in Section 4.

\section{Theory of PR}

\subsection{The Principle of $P R$}

PR technology is based on the theory that the diffraction of coherent light propagates. PR generally involves estimating a complex-valued phase distribution from known intensity distributions at some properly selected planes. It is an inverse problem in optics, which uses the Fourier transform relationship between the pupil and the in-focus plane to iteratively estimate the phase which is suffering from non-uniqueness. Figure 1 shows the schematic layout of the PR principle. When a beam propagates along the optical axis, the diffraction field distribution is formed at a certain propagation distance. The reference wave emitted from the light source is incident on the measured mirror. After the reflection of the output light field, the complex amplitude distribution of the optical wave front contains the error information of the measured mirror. Using these intensity images and the PR algorithm, one can accurately recover the surface error of the measured mirror [20]. The detector is placed at the focal plane of the wave front reflected or transmitted from the surface under testing and will take a number of images, including those in focus and defocused from the focal plane in both directions. The wave front can be estimated with the known pupil size and the defocus amounts of the detectors [21].

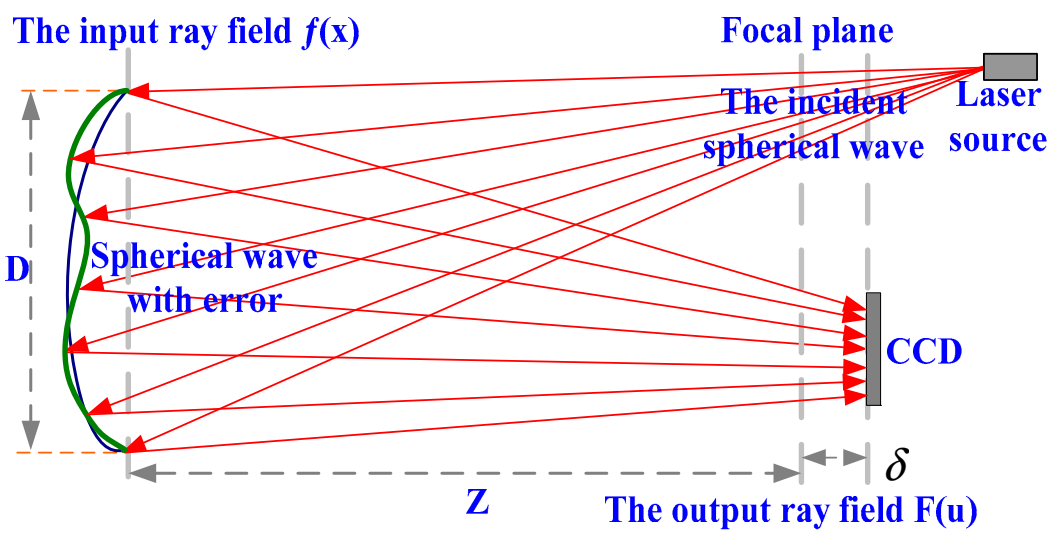

Figure 1. The principle of the Phase Retrieval system.

Assuming that the aperture of one measured optical surface is D, the focal length is $Z$, and the laser wavelength is $\lambda$. The generalized pupil function is $f(x)$, whose amplitude is $|f(x)|$, and the phase is $\theta$, which can be obtained with Zernike polynomial fitting: $\theta(x)=\sum_{n} \alpha_{n} Z_{n}(x)$, where the real number $\alpha_{n}$ represents the first $n$th terms of the polynomial coefficients and $Z_{n}$ indicates the $n$th terms of the Zernike polynomials.

We could get:

$$
f(x)=|f(x)| \exp [i \theta(x)]
$$

where $x$ is an $\mathrm{M}$-dimensional spatial coordinate, and $\theta$ is wave-front distortion. 
For a linear optical system with the defocus $\delta$ in the focal plane, the impulse response function $F(u)$ can be described as:

$$
F(u)=|F(u)| \exp [i \psi(u)]=\mathcal{F}\{|f(x)| \exp [\varepsilon(x, \delta)]\}
$$

where $x$ is the spatial coordinate of the pupil, $u$ is the coordinates of the image and both of them are two-dimensional vector field coordinates. $\psi$ is the phase of the impulse response, $\mathcal{F}$ is the two-dimensional Fourier transform, and $\varepsilon(x, \delta)$ is the wave-front aberration caused by the defocus $\delta$.

For one PR system, $|f(x)|$ in Equation (1) is the priori condition of a known optical system corresponding to the size and shape of the pupil. $|F(u)|^{2}$ is the image collected by the detector. We estimate $\alpha_{n}$ and then obtain $\theta$ in Equation (1) with a number of measurements at different defocuses.

\subsection{The Modified Gradient Search Algorithm of PR}

In this paper, we present a modified gradient search algorithm to solve the phase recovery problem $[22,23]$. Let $g_{m, k}, \theta_{m, k}, G_{m, k}, \varphi_{m, k}$ be the estimated values of $f, \delta, F, \psi$ when the $m$ th images iterate $k$ times, $g_{k}$ represents the combined estimate value with every $g_{m, k}$ to $f$ when iterated $k$ times, which is $g_{k}(x)=\frac{1}{M} \sum_{m=1}^{M} g_{m, k}(x)$.

The initialization is:

$$
\begin{array}{ll}
\text { a. } & \theta_{m, k}=0, \varepsilon_{m}(x)=\varepsilon\left(x, \delta_{m}\right)=\left(\frac{\pi \delta_{m}\|x\|^{2}}{\lambda Z^{2}}\right), g_{k}(x)=|f(x)|, \mathrm{m} \in[1, \mathrm{M}], \\
\text { b. } & G_{m, k}(u)=\left|G_{m, k}(u)\right| \exp \left[i \varphi_{m, k}(u)\right]=\mathcal{F}\left\{g_{k}(x) \exp \left[i \varepsilon_{m}(x)\right]\right\}, \mathrm{m} \in[1, \mathrm{M}], \\
\text { c. } & G_{m, k^{\prime}}(u)=|F(u)| \exp \left[i \varphi_{m, k}(u)\right], \mathrm{m} \in[1, \mathrm{M}], \\
\text { d. } & g_{m, k}(x)=\left|g_{m, k}(x)\right| \exp \left[i \theta_{m, k^{\prime}}(x)\right]=\mathcal{F}^{-1}\left[G_{m, k}(u)\right] \exp \left[-\varepsilon_{m}(x)\right], \mathrm{m} \in[1, \mathrm{M}], \\
\text { e. } & g_{m, k+1}(x)=|f(x)| \exp \left[i \theta_{m, k+1}(x)\right]=|f(x)| \exp \left[i \theta_{m, k^{\prime}}(x)\right], \mathrm{m} \in[1, \mathrm{M}], \\
\text { f. } & g_{k+1}(x)=\frac{1}{M} \sum_{m=1}^{M} g_{m, k+1}(x) .
\end{array}
$$

Repeat from steps $b$ to steps $f$ until the extrusion of the condition, which is the limitation of the iteration times or the function of the object descended to the appointed value. The function of the object is described as [24]:

$$
B_{k}=E_{F K}^{2}=N^{-2} \sum_{m=1}^{M} \sum_{u}\left|G_{m, k}(u)-G_{m, k}{ }^{\prime}(u)\right|^{2}
$$

where $N$ represents the width of the collected images. According to $\mathrm{b}$ and $\mathrm{c}$, the phase $G_{m, k}(u)$ and the phase $G_{m, k}{ }^{\prime}(u)$ are equal, so we can get:

$$
B_{k}=E_{F K}^{2}=N^{-2} \sum_{m=1}^{M} \sum_{u}\left[\left|G_{m, k}(u)\right|-|F(u)|\right]^{2}
$$

We apply the mathematical optimization method with Equation (3) as the function of the object and the unknown quantity about each partial derivative together with the substitution gradient search algorithm, finally obtaining the estimation of the wave-front distortion corresponding to $\theta$, when $B_{k}$ is smallest. The most important application of the gradient search algorithm is the correct description of the function of the object and the partial derivatives of each variable. We first discuss the partial derivative $g(x)$, which is as the unknown variables. We get the derivative from $B$ to $g(x)$, respectively, and get the derivative from $B_{k}$ to the real part of $\partial g_{r e a l}$ and the imaginary part of $\partial g_{i m a g}$

$$
\begin{gathered}
\partial g_{\text {real }} B_{k} \equiv \frac{\partial B_{k}}{\partial g_{\text {real. } k}(x)}=2 N^{-2} \sum_{m=1}^{M} \sum_{u}\left[\left|G_{m, k}(u)\right|-|F(u)|\right] \frac{\partial\left|G_{m, k}(u)\right|}{\partial g_{\text {real }, k}(x)} \\
\partial g_{\text {imag }} B_{k} \equiv \frac{\partial B_{k}}{\partial g_{\text {imag. } k}(x)}=-i 2 N^{-2} \sum_{m=1}^{M} \sum_{u}\left[\left|G_{m, k}(u)\right|-|F(u)|\right] \frac{\partial\left|G_{m, k}(u)\right|}{\partial g_{\text {imag }, k}(x)}
\end{gathered}
$$


where

$$
\begin{aligned}
& \frac{\partial\left|G_{m, k}(u)\right|}{\partial g_{\text {real }, k}(x)}=\frac{\partial}{\partial g_{\text {real }, k}(x)} \sum_{y} g_{k}(y) \exp \left[i \varepsilon_{m}(x)\right] \exp \left[\frac{-i 2 \pi u y}{N}\right]=\exp \left[i \varepsilon_{m}(x)\right] \exp \left[\frac{-i 2 \pi u x}{N}\right] \\
& \frac{\partial\left|G_{m, k}(u)\right|}{\partial g_{\text {imag }, k}(x)}=\frac{\partial}{\partial g_{\text {imagg }, k}(x)} \sum_{y} g_{k}(y) \exp \left[i \varepsilon_{m}(x)\right] \exp \left[\frac{-i 2 \pi u y}{N}\right]=\exp \left[i \varepsilon_{m}(x)\right] \exp \left[\frac{-i 2 \pi u x}{N}\right]
\end{aligned}
$$

and

$$
\begin{aligned}
& \frac{\partial\left|G_{m, k}(u)\right|}{\partial g_{\text {real }, k}(x)}=\frac{\partial\left[\left|G_{m, k}(u)\right|^{2}\right]^{1 / 2}}{\partial g_{\text {real }, k}(x)}=\frac{1}{2\left|G_{m, k}(u)\right|} \frac{\partial\left|G_{m, k}(u)\right|^{2}}{\partial g_{\text {real }, k}(x)}=\frac{G(u) \exp \left[-i \varepsilon_{m}(x)+i 2 \pi u x / N\right]}{2|G(u)|}+\text { c.c. } \\
& \frac{\partial\left|G_{m, k}(u)\right|}{\partial g_{\text {imag }, k}(x)}=\frac{\partial\left[\left|G_{m, k}(u)\right|^{2}\right]^{1 / 2}}{\partial g_{\text {imag }, k}(x)}=\frac{1}{2\left|G_{m, k}(u)\right|} \frac{\left.\partial G_{m, k}(u)\right|^{2}}{\partial g_{\text {imag }, k}(x)}=\frac{G(u) \exp \left[-i \varepsilon_{m}(x)+i 2 \pi u x / N\right]}{2|G(u)|}+\text { c.c. }
\end{aligned}
$$

Thus, Equation (4) can be changed to:

$$
\begin{gathered}
\partial g_{\text {real }} B_{k}=N^{-2} \sum_{m=1}^{M} \sum_{u}\left[G_{m, k}(u)-|F(u)| G_{m, k}(u) /\left|G_{m, k}(u)\right|\right]=\frac{-i G(u) \exp \left[-i \varepsilon_{m}(x)+i 2 \pi u x / N\right]}{2|G(u)|}+\text { c.c. } \\
\partial g_{\text {imag }} B_{k}=-i N^{-2} \sum_{m=1}^{M} \sum_{u}\left[G_{m, k}(u)-|F(u)| G_{m, k}(u) /\left|G_{m, k}(u)\right|\right]=\frac{-i G(u) \exp \left[-i \varepsilon_{m}(x)+i 2 \pi u x / N\right]}{2|G(u)|}+\text { c.c. }
\end{gathered}
$$

where c.c. represents the former plural conjugate.

Using $G_{m, k}{ }^{\prime}(u)=|F(u)| \exp \left[i \phi_{m, k}(u)\right], m \in[1, M]$ to define $G_{m, k^{\prime}}(u)$, we could get: $G_{m, k^{\prime}}(u)=\frac{\left|F(u) G_{m, k}(u)\right|}{\left|G_{m, k}(u)\right|}$.

Thus, Equation (7) can be expressed as:

$$
\begin{aligned}
\partial g_{\text {real }} B_{k} & =2 \operatorname{Real} \sum_{m}\left[g_{m, k}(x)-g_{m, k^{\prime}}(x)\right] \\
\partial g_{\text {imag }} B_{k} & =2 \operatorname{Imag} \sum_{m}\left[g_{m, k}(x)-g_{m, k^{\prime}}(x)\right]
\end{aligned}
$$

We consider $\theta(x)$ as the derivative of the unknown value. From Equation (3) we get the derivative from $B_{k}$ to $\theta(x)$ :

$$
\partial_{\theta} B_{k}=\frac{\partial B_{k}}{\partial \theta_{k}(x)}=2 N^{-2} \sum_{m} \sum_{u}\left[\left|G_{m, k}(u)\right|-|F(u)|\right] \frac{\partial\left|G_{m, k}(u)\right|}{\partial \theta_{k}(x)}
$$

Because of

$$
\frac{\partial\left|G_{m, k}(u)\right|}{\partial \theta_{k}(x)}=\frac{\partial}{\partial \theta_{k}(x)} \sum_{y}|f(y)| \exp [i \theta(y)] \exp \left[i \varepsilon_{m}(x)\right] \exp \left[\frac{-i 2 \pi u y}{N}\right]=i g_{k}(x) \exp \left[i \varepsilon_{m}(x)\right] \exp \left[\frac{-i 2 \pi u x}{N}\right]
$$

Then, we could get:

$$
\frac{\partial\left|G_{m, k}(u)\right|}{\partial \theta_{k}(x)}=\frac{G_{m, k}(u)(-i) g_{k}^{*}(x) \exp \left[-i \varepsilon_{m}(x)\right] \exp [i 2 \pi u x / N]+\text { c.c. }}{2\left|G_{m, k}(u)\right|}
$$

Thus, we could get:

$$
\begin{aligned}
\partial_{\theta} B_{k} & =\sum_{m} i g_{m, k^{*}}(x)\left[g_{m, k^{\prime}}(x)-g_{m, k}(x)\right]+\text { c.c. } \\
& =-2 \operatorname{Imag} \sum_{m}\left[g_{m, k}{ }^{*}(x) g_{m, k^{\prime}}(x)\right] \\
& =-2|f(x)| \sum_{m}\left|g_{m, k^{\prime}}(x)\right| \sin \left[\theta_{m, k^{\prime}}(x)-\theta_{m, k}(x)\right]
\end{aligned}
$$


We consider the Zernike coefficient $\alpha(x)$ as the derivation of the unknown value. From Equation (3), we get the derivative from $B_{k}$ to $\alpha(x)$ :

$$
\frac{\partial B_{k}}{\partial \alpha_{n, k}}=\sum_{x} \frac{\partial B}{\partial \theta_{k}(x)} \frac{\partial \theta_{k}(x)}{\partial \alpha_{n, k}(x)}
$$

Take $\frac{\partial \theta_{k}(x)}{\partial \alpha_{n, k}(x)}=\frac{\partial}{\partial \alpha_{n, k}}\left[\sum_{n=1}^{m} \alpha_{n, k} Z_{n}(x)\right]=Z_{n}(x)$ into Equation (12). We get the objective function, which is calculated as:

$$
\partial_{\alpha_{n}} B_{k}=-2 \sum_{m} \sum_{x}|f(x)|\left|g_{m, k^{\prime}}(x)\right| \sin \left[\theta_{m, k^{\prime}}(x)-\theta_{m, k}(x)\right] Z_{n}(x)
$$

With the objective Equation (3) and its impact on the Zernike coefficient derivative Equation (13), we can use the mathematical optimization algorithm, such as Limitedmemory BFGS algorithm, to solve various Zernike wave-front coefficient values [25-27].

\section{Experimental Demonstrations}

Here, we demonstrate the measurement ability in small-slope free-form surfaces with the modified phase retrieval discussed in Section 2. Figure 2a shows the diagram of the measuring installation with the course of the clearance beams. In addition, we built the experimental setup to measure the thin, deformable mirror surface (in Figure 2c), shown in Figure $2 \mathrm{~b}$. The size of this measured mirror is (35 mm (length) $\times 35 \mathrm{~mm}$ (width) $\times 15 \mathrm{~mm}$ (thickness)), and there are three screws on the back surface of mirror which were used to apply different forces in order to change the surface shape. The collimated laser beam (with the wavelength of $632.5 \mathrm{~nm}$ ) from the WYKO interferometer passed through the beam splitter and reached the measured surface. The reflected light from the measured surface was directed to the focusing lens, with a focal length of $150 \mathrm{~mm}$, by the beam splitter and then reached the detector. The detector was placed on a computerized moving stage which enabled the detector to take images as it moved away from the focal plane.

In the experiment, the beam size was limited to $10 \mathrm{~mm}$ by a stop. Firstly, we built the experiment system, fixed the thin measured mirror in the stage, observed the fringes in WYKO interferometer to maximize the contrast of the interference fringes, added the splitting prism in the optical path, and adjusted them to be coaxial with the measured mirror and pinhole. Secondly, we adjusted the position of the lens and the camera so that the light beam reflected from the measured surface through the prism and entered into the camera. We captured seven images with the camera, and the defocus amounts were $0, \pm 1.2, \pm 1.7$, and $\pm 2.2 \mathrm{~mm}$. Thirdly, we disposed the collected images with the modified PR algorithm to obtain the mirror surface information. In order to make an effective comparison with the WYKO interferometer, we did not move the position of the experimental setup and measured the deformed surface again. We first measured a reference mirror with $1 / 20$ wave flatness to remove the system errors. The measurement data with the flat mirror are treated as the system errors and subtracted from them when measuring the free-form surfaces. The seven images with different defocuses solved by modified PR are shown in Figure 3a. Figure 3b shows the estimated surface shape recovered by the modified PR with the two images in Figure 3a. The process of obtaining Figure $3 b$ took $5.50 \mathrm{~s}$, and Figure 3c shows the estimated surface shape recovered by the modified PR with all seven images. The process of obtaining Figure $3 \mathrm{c}$ took $75.35 \mathrm{~s}$, which means that the improved PR algorithm was 15 times as fast. Figure $3 \mathrm{~d}$ shows the difference between the estimated surface shapes recovered by the modified PR with all seven images and the two images. We could see that the difference was very small. This experiment demonstrates that the proposed modified PR algorithm is feasible in the surface metrology. 


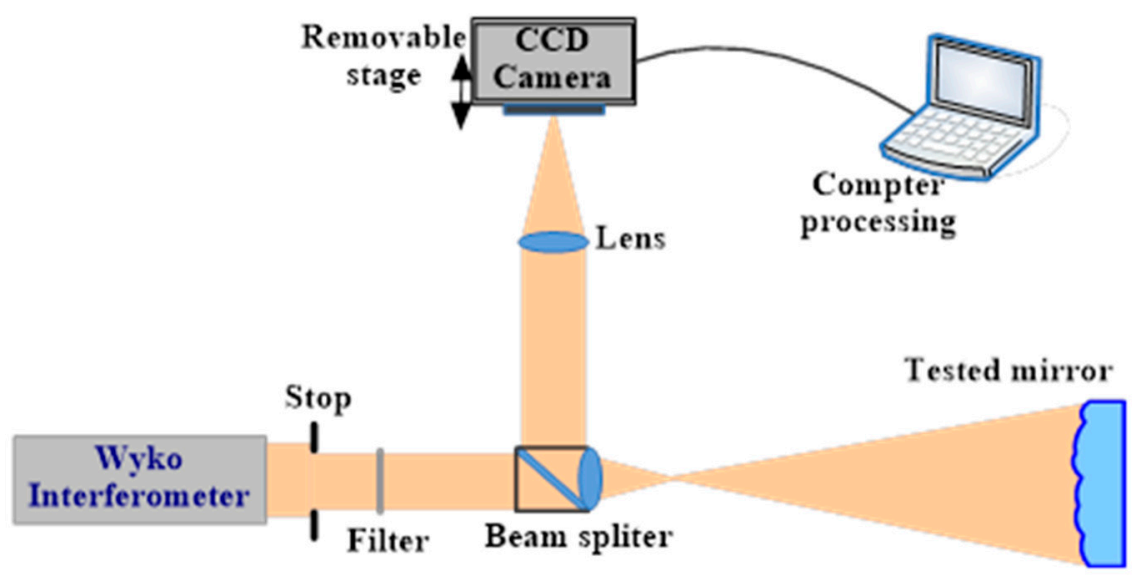

(a)

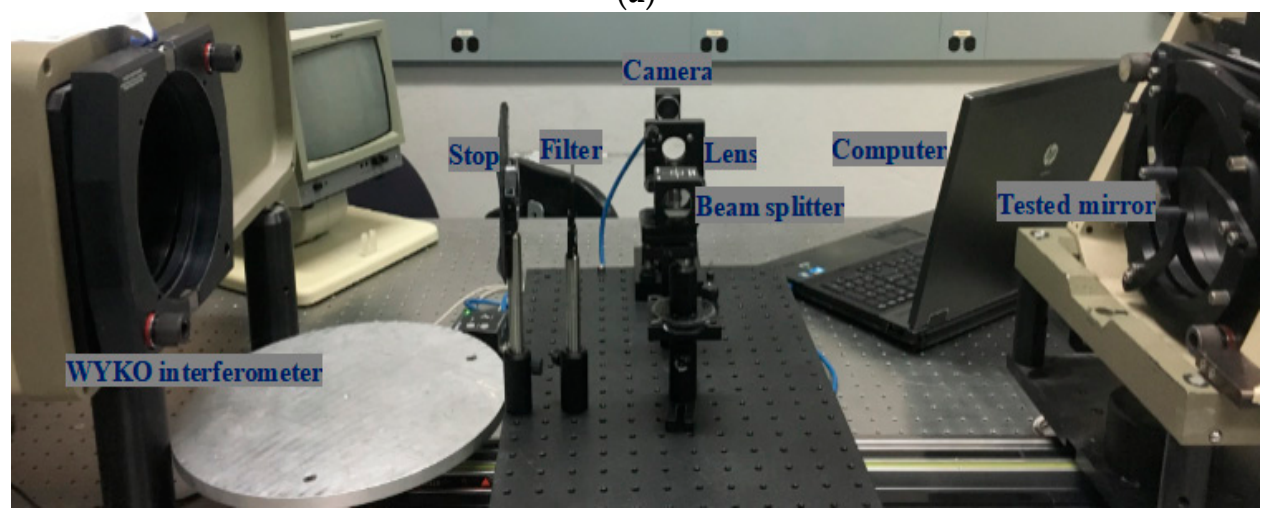

(b)

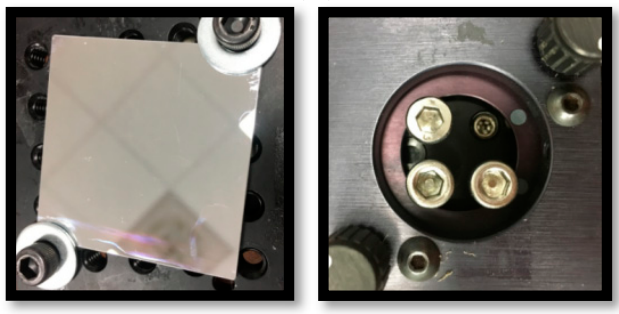

(c)

Figure 2. (a) The diagram of the measuring installation with the course of the clearance beams; (b) the experimental setup of the PR system; and (c) the thin measured mirror. The left is the front surface of the thin measured mirror and the right is the back surface of the thin measured mirror. Three screws were used to apply different forces to the measured mirror to change the surface shape.

To demonstrate the feasibility of the proposed method in free-form surface metrology, we apply different forces to the thin mirror, shown in Figure 2c, and take two defocused images for each force to estimate the free-form mirror surface with the improved PR algorithm. Figure $4 a, b$ are, respectively, the mirror surface estimated by PR and the mirror surface measured with the WYKO interferometer. Comparing the results of the modified PR with the results of WYKO interferometer, the RMS difference is less than $2.777 \mathrm{~nm}$, which shows that the proposed improved PR method is feasible for measuring free-form surfaces. The difference in the PV is relatively large, partially due to the following reasons. Firstly, there is a smoothing process when using the WYKO interferometer, which the solution process of the modified PR method does not have. Secondly, during the solution process we calculated the whole mask circular area with the modified PR, but Figure $4 \mathrm{~b}$ was obtained after matting (removing boundary Burr) with the WYKO interferometer. Therefore, although the RMS of the whole mask cannot be greatly affected, it will be greatly different from the PV. 


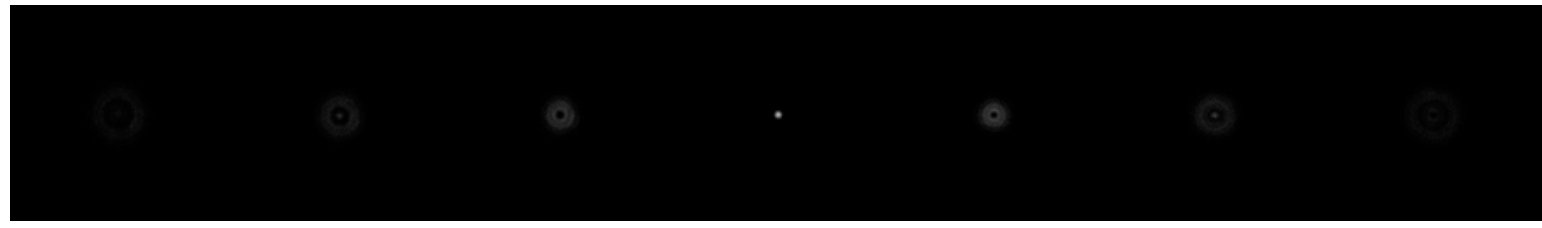

(a)

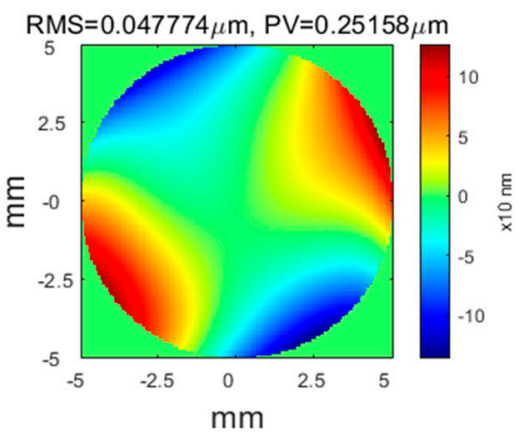

(b)

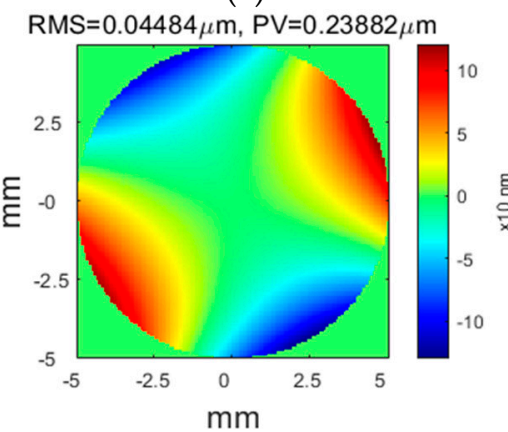

(c)

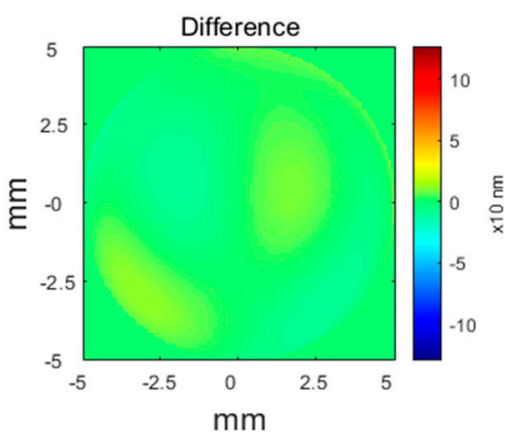

(d)

Figure 3. (a) Seven 128 pixels * 128 pixels defocused images, from left to right, the defocus is, respectively, $-2.2 \mathrm{~mm},-1.7 \mathrm{~mm},-1.2 \mathrm{~mm}, 0 \mathrm{~mm}, 1.2 \mathrm{~mm}, 1.7 \mathrm{~mm}$, and $2.2 \mathrm{~mm}$; (b) the solved result of the estimated surface shape with two images in (a) (the defocus amounts are $-1.7 \mathrm{~mm}$ and $1.7 \mathrm{~mm}$, respectively) is RMS $=0.047774 \mu \mathrm{m}$ and PV $=0.25158 \mu \mathrm{m}$; (c) the solved result of the estimated surface shape with all seven images in (a) is RMS $=0.04484 \mu \mathrm{m}$ and PV $=0.23882 \mu \mathrm{m}$; and (d) the difference between the measurement results with two images and with all seven images.

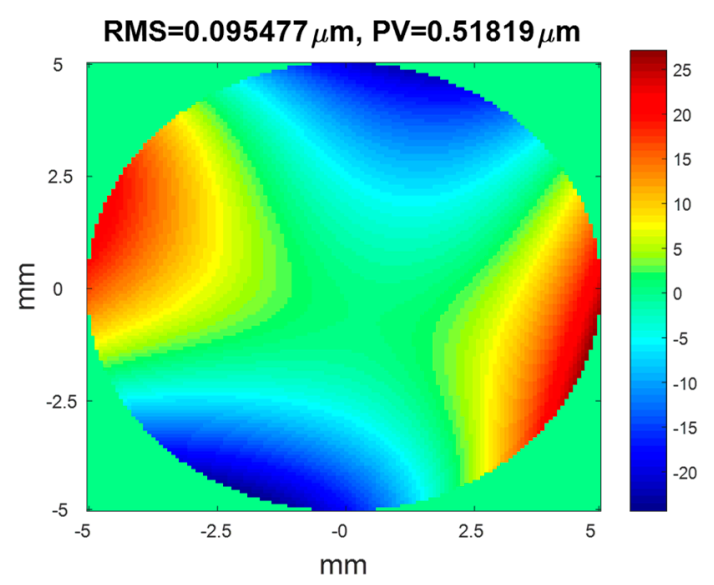

(a)

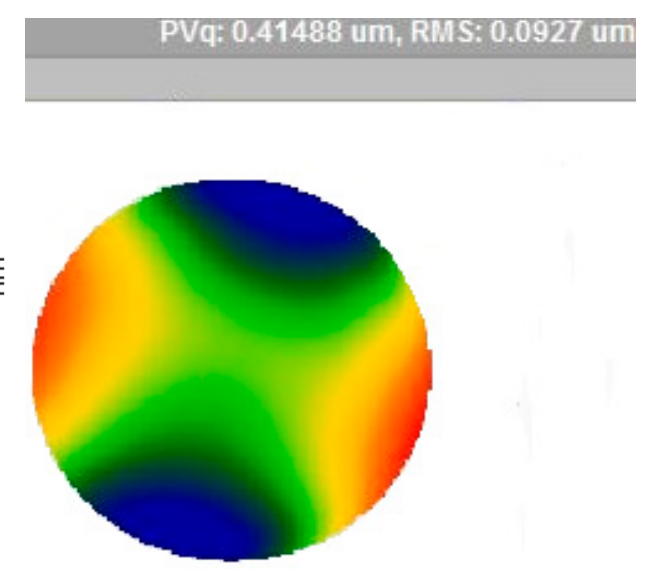

(b)

Figure 4. The measurement results with (a) PR: RMS $=0.095477 \mu \mathrm{m}, \mathrm{PV}=0.51819 \mu \mathrm{m}$; (b) WYKO: RMS $=0.0927 \mu \mathrm{m}, \mathrm{PV}=0.41488 \mu \mathrm{m}$.

It can be seen from the above two experimental results that the process of obtaining Figure $3 b$ from the two images took $5.50 \mathrm{~s}$ and the process of obtaining Figure $3 \mathrm{c}$ with all seven images took $75.35 \mathrm{~s}$, which means that the improved PR algorithm is 15 times faster. Besides, Figure $4 a, b$, respectively, show the estimated surface recovered by PR and the measured surface with the WYKO interferometer; the differences between our technique and the WYKO interferometer in RMS and PV are very small, which demonstrated that our improved PR method could achieve as considerable an accuracy as the WYKO interferometer. The above two points proved the feasibility and effectiveness of our technology in the measurement of small-slope free-form surfaces. 


\section{Conclusions}

In this paper, we have presented and shown experimentally with an improved PR algorithm based on the traditional gradient search algorithm to improve efficiency of phase recovery. The feasibility of the proposed method has been demonstrated by comparing the measurement results of the deformed thin mirror with the measurement results from WYKO interferometer. This work has additionally shown that PR technology is a viable and realistic method in small-slope free-form surfaces measurement. Now, we are doing research on large-slope free-form surfaces measurement with transverse translation diversity phase retrieval, and our new research will perhaps be shown in the near future.

Author Contributions: Conceptualization, X.M.; methodology, X.M. and J.W.; software, B.W. and X.M.; validation, J.W. and X.L.; formal analysis, X.M. and X.L.; investigation, X.M., J.W. and B.W.; resources, X.M. and B.W.; data curation, X.M.; writing—original draft preparation, X.M.; writingreview and editing, X.L.; supervision, J.W.; funding acquisition, X.M. All authors have read and agreed to the published version of the manuscript.

Funding: This work was supported by the Program of Chinese Academy of Sciences Scholarship Council (201608290106) and the National Natural Science Foundation of China (NSFC) (Grant No. 62105327).

Data Availability Statement: Not applicable.

Acknowledgments: The authors would like to thank Rongguang Liang from the College of Optical Sciences, University of Arizona, for providing the experiment platform and academic guidance.

Conflicts of Interest: The authors declare no conflict of interest related to this article.

\section{References}

1. Ye, J.; Chen, L.; Li, X.; Yuan, Q.; Gao, Z. Review of optical freeform surface representation technique and its application. Opt. Eng. 2017, 56, 110901. [CrossRef]

2. Wei, L.; Li, Y.; Jing, J.; Feng, L.; Zhou, J. Design and fabrication of a compact off-axis see-through head-mounted display using a freeform surface. Opt. Express 2018, 26, 8550-8565. [CrossRef] [PubMed]

3. Wei, S.L.; Zhu, Z.B.; Fan, Z.C.; Yan, Y.M.; Ma, D.L. Multi-surface catadioptric freeform lens design for ultra-efficient off-axis road illumination. Opt. Express 2019, 27, A779-A789. [CrossRef]

4. Meng, Q.; Wang, W.; Ma, H.; Dong, J. Easy-aligned off-axis three-mirror system with wide field of view using freeform surface based on integration of primary and tertiary mirror. Appl. Opt. 2014, 53, 3028-3034. [CrossRef] [PubMed]

5. Wang, Q.; Cheng, D.; Wang, Y.; Hua, H.; Jin, G. Design, tolerance, and fabrication of an optical see-through head-mounted display with free-form surface elements. Appl. Opt. 2013, 52, C88-C99. [CrossRef] [PubMed]

6. Zhu, R.H.; Sun, Y.; Shen, H. Progress and Prospect of Optical Freeform Surface Measurement. Acta Opt. Sin. 2021, 41, 0112001.

7. Pant, K.K.; Burada, D.R.; Bichra, M.; Singh, M.P.; Ghosh, A.; Khan, G.S.; Sinzinger, S.; Shakher, C. Subaperture stitching for measurement of freeform wavefront. Appl. Opt. 2015, 54, 10022-10028. [CrossRef] [PubMed]

8. El-Hayek, N.; Nouira, H.; Anwer, N.; Damak, M.; Gibaru, O. Reconstruction of freeform surfaces for metrology. J. Phys. Conf. Ser. 2014, 483, 012003. [CrossRef]

9. DeFisher, S.; Bechtold, M.; Mohring, D. A non-contact surface measurement system for freeform and conformal optics. Proc. SPIE 2011, 8016, 80160W. [CrossRef]

10. Ma, X.; Wang, J. Spherical mirror testing by phase retrieval wavefront sensor. Optik 2016, 127, 2396-2400. [CrossRef]

11. Michalko, A.M.; Fienup, J.R. Sensitivity study of transverse translation diverse phase retrieval for freeform metrology. In Optical Manufacturing and Testing XII; International Society for Optics and Photonics: Bellingham, WA, USA, 2018; Volume 10742, p. 107420T.

12. Moore, D.B.; Fienup, J. Subaperture translation estimation accuracy in transverse translation diversity phase retrieval. Appl. Opt. 2016, 55, 2526-2536. [CrossRef]

13. Zeng, F.; Tan, Q.; Liu, Y.; Gu, H.; Zhou, Z.; Jin, G. Freeform metrology based on phase retrieval and computer-generated hologram. In Optical Design and Testing VI; International Society for Optics and Photonics: Bellingham, WA, USA, 2014; Volume 9272, p. 92720E.

14. Fienup, J.; Marron, J.C.; Schulz, T.J.; Seldin, J.H. Hubble Space Telescope characterized by using phase-retrieval algorithms. Appl. Opt. 1993, 32, 1747-1767. [CrossRef] [PubMed]

15. Dean, B.H.; Aronstein, D.L.; Smith, J.S.; Shiri, R.; Acton, D.S. Phase retrieval algorithm for JWST flight and testbed telescope. In Space Telescopes and Instrumentation I: Optical, Infrared, and Millimeter; International Society for Optics and Photonics: Bellingham, WA, USA, 2006; Volume 6265, p. 626511. 
16. Brady, G.R.; Guizar-Sicairos, M.; Fienup, J. Optical wavefront measurement using phase retrieval with transverse translation diversity. Opt. Express 2009, 17, 624-639. [CrossRef]

17. Wu, Y.; Ding, L.; Hu, X. An improved phase retrieval algorithm for optical aspheric surface measurement. Opt. Commun. 2011, 284, 1496-1503. [CrossRef]

18. Feng, Z.; Cheng, D.; Wang, Y. Transferring freeform lens design into phase retrieval through intermediate irradiance transport. Opt. Lett. 2019, 44, 5501-5504. [CrossRef]

19. Michalko, A.M.; Fienup, J.R. Concave Mirror Measurement Using Transverse Translation Diverse Phase Retrieval. In Optical Fabrication and Testing; Optical Society of America: Washington, DC, USA, 2017; p. OW2B.5.

20. Jurling, A.S.; Fienup, J.R. A numerical exploration of phase-retrieval error-metric surfaces. In Adaptive Optics System IV; International Society for Optics and Photonics: Bellingham, WA, USA, 2014; Volume 9148, p. 91486N.

21. Moore, D.B.; Fienup, J.R. Extending the capture range of phase retrieval through random starting parameters. In Frontiers in Optics; OSA Technical Digest; Optical Society of America: Washington, DC, USA, 2014; p. FTu2C.2.

22. Jurling, A.S.; Fienup, J.R. Applications of algorithmic differentiation to phase retrieval algorithms. J. Opt. Soc. Am. A 2014, 31, 1348-1359. [CrossRef]

23. Lim, S.; Shin, J. Application of a Deep Neural Network to Phase Retrieval in Inverse Medium Scattering Problems. Computation 2021, 9, 56. [CrossRef]

24. Ma, X.; Wang, J. The basic research of phase retrieval algorithm. Optik 2016, 127, 1561-1566. [CrossRef]

25. Jurling, S.; Fienup, J.R. Phase retrieval with unknown sampling factors via the two-dimensional chirp-Z transform. J. Opt. Soc. Am. A 2014, 31, 1904-1911. [CrossRef]

26. Tsai, Y.-J.; Bousse, A.; Ehrhardt, M.J.; Stearns, C.W.; Ahn, S.; Hutton, B.F.; Arridge, S.; Thielemans, K. Fast Quasi-Newton Algorithms for Penalized Reconstruction in Emission Tomography and Further Improvements via Preconditioning. IEEE Trans. Med. Imaging 2018, 37, 1000-1010. [CrossRef] [PubMed]

27. Aşırım, Ö.E.; Yolalmaz, A.; Kuzuoğlu, M. High-Fidelity Harmonic Generation in Optical Micro-Resonators Using BFGS Algorithm. Micromachines 2020, 11, 686. [CrossRef] [PubMed] 